\title{
Protective Effect of Shexiang Baoxin Pill on Myocardial Ischemia/ Reperfusion Injury in Patients With STEMI
}

\author{
Haixia Qin, Siyuan Li and Zhenbing Liu* \\ Ordos Central Hospital, Ordos Clinical Medical College, Inner Mongolia Medical University, Ordos, China
}

Background: There is no definite effect in the treatment of myocardial ischemia/ reperfusion (I/R) injury in patients with acute ST-segment elevation myocardial infarction (STEMI). We evaluated the protective effect of Shexiang Baoxin Pill (SBP) on I/R injury in STEMI patients.

Methods: STEMI patients were randomly divided into a primary percutaneous coronary intervention (PPCl) group $(n=52)$ and a PPCl + SBP group $(n=51)$. The area at risk of infarction (AAR) and final infarct size (FIS) were examined by single-photon emission

OPEN ACCESS

Edited by:

Hua Zhu,

The Ohio State University,

United States

Reviewed by:

Beat M. Jucker,

GlaxoSmithKline, United States

Paola Signorelli,

University of Milan, Italy

${ }^{*}$ Correspondence:

Zhenbing Liu

LZHBL44@126.COM

Specialty section:

This article was submitted to

Cardiovascular and Smooth Muscle

Pharmacology,

a section of the journal

Frontiers in Pharmacology

Received: 05 June 2021

Accepted: 25 August 2021

Published: 10 September 2021

Citation:

Qin H, Li S and Liu Z (2021) Protective Effect of Shexiang Baoxin Pill on Myocardial Ischemia/Reperfusion

Injury in Patients With STEMI.

Front. Pharmacol. 12:721011.

doi: 10.3389/fphar.2021.721011 computed tomography (SPECT). I/R injury was assessed using myocardial salvage (MS) and salvage index (SI) calculated from AAR and FIS.

Results: The ST-segment resolution (STR) in the PPCl + SBP group was significantly higher than that in the PPCl group ( $p=0.036)$, and the peak value of high-sensitivity troponin T (hsTNT) was lower than that in the PPCl group $(p=0.048)$. FIS in the $\mathrm{PPCl}+$ SBP group was smaller than that in the PPCl group $(p=0.047)$. MS $(p=0.023)$ and $\mathrm{SI}(p=$ 0.006) in the $\mathrm{PPCl}+\mathrm{SBP}$ group were larger than those in the PPCl group. The left ventricular ejection fraction (LVEF) in the PPCl + SBP group was higher than that in the $\mathrm{PPCl}$ group ( $D=0.049$ ), and $\mathrm{N}$-terminal pro-B type natriuretic peptide (NT-proBNP) level in the $\mathrm{PPCl}+\mathrm{SBP}$ group was lower than that in the PPCl group $(p=0.048)$.

Conclusions: SBP can alleviate I/R injury (MS and SI), decrease myocardial infarction area (peak value of hsTNT and FIS), and improve myocardial reperfusion (MBG and STR) and cardiac function (LVEF and NT-proBNP).

Keywords: shexiang baoxin pill, ST-segment elevation myocardial infarction, myocardial ischemia reperfusion injury, myocardial protection, myocardial salvage

Abbreviations: AAR, area at risk of infarction; ACEI, angiotensin-converting enzyme inhibitor; ADAC, automatic direct analog computer; ARB, angiotensin receptor blockers; AMI, acute myocardial infarction; CHD, coronary heart disease; CK-MB, creatine kinase-myocardial band; cTNT, cardiac troponin T; cTNI, cardiac troponin I; ECG, electrocardiogram; FIS, final infarct size; hsTNT, high-sensitivity troponin T; I/R, ischemia/reperfusion; LVEF, left ventricular ejection fraction; MACCE main adverse cardiovascular events; MBG, myocardial blush grade; MPTP, mitochondrial permeability transition pore; MS, myocardial salvage; NSTE-ACS, Non-ST-elevation acute coronary syndromes; NT-proBNP, N-terminal pro-B type natriuretic peptide; NT-proBNP, N-terminal pro-B type natriuretic peptide; PPCI, primary percutaneous coronary intervention; QPS, quantitative perfusion software; RCTs, randomized controlled trials; RIC, remote ischemic conditioning; SI, salvage index; STR, ST-segment resolution; SPECT, single-photon emission computed tomography; STEMI, ST-segment elevation myocardial infarction; TIMI, Thrombolysis in myocardial infarction. 


\section{INTRODUCTION}

ST-segment elevation myocardial infarction (STEMI) is the main cause of death in patients with coronary heart disease (CHD), and its incidence is increasing (GBD 2015 Disease and Injury Incidence and Prevalence Collaborators, 2018). With the continuous progress of various treatment measures including reperfusion treatment, the mortality of STEMI patients has been significantly reduced (Bulluck et al., 2016). However, reperfusion treatment can induce myocardial ischemia/reperfusion (I/R) injury Hausenloy and Yellon (2015), which can result in poor prognosis (Bolognese et al., 2004). Therefore, effective treatment of $\mathrm{I} / \mathrm{R}$ injury is critical. At present, no measures have been found for effective treatment of $\mathrm{I} / \mathrm{R}$ injury in STEMI patients (Hausenloy et al., 2017). The pathogenesis of $I / R$ injury is diverse Hausenloy et al. (2017), and cooperative treatment through different mechanisms may be a new approach for I/R injury treatment (Davidson et al., 2019). Shexiang Baoxin Pill (SBP) is a traditional Chinese medicine compound preparation, it is mainly composed of artificial moschus, radix ginseng, calculus bovis artifactus, cortex cinnamomi, styrax, venenum bufonis, and borneolum syntheticum, and is mainly used to treat CHD (Lu et al., 2018; Ge et al., 2021). The extract of the components of SBP reduces $\mathrm{I} / \mathrm{R}$ injury, myocardial remodeling, and myocardial infarction area in animals through multiple mechanisms. These multiple mechanisms of action may provide a basis for the use of SBP in the treatment of STEMI (Wu et al., 2011; Fancelli et al., 2014; Zheng et al., 2017; Du et al., 2018a; Du et al., 2018b; Li et al., 2018; Li et al., 2020). Recent animal studies have found that SBP can reduce I/R injury and final infarct size (FIS) (Xiang et al., 2013). Small clinical trials with low methodological quality and ill-defined bias risk have demonstrated that SBP decreased the level of myocardial injury markers and main adverse cardiovascular events (MACCE) in patients with acute myocardial infarction (AMI) (Lu et al., 2018). SBP added to conventional treatment may have beneficial effects on the longterm outcomes of Non-ST-elevation acute coronary syndromes (NSTE-ACS) (Zhou et al., 2016). Therefore, SBP may reduce myocardial I/R injury and myocardial infarction area in human patients. Thus, the current study was designed to examine the therapeutic effect of SBP in I/R injury of STEMI patients.

\section{MATERIALS AND METHODS}

\section{General Information}

From September 1, 2016 to March 31, 2019, 110 patients with STEMI in the Second District of Cardiology Department of Ordos Central Hospital were selected to receive primary percutaneous coronary intervention (PPCI). The inclusion criteria included: age $\geq 18$ years; chest pain $<12 \mathrm{~h}$; and the first electrocardiogram (ECG) showed ST segment elevation $\geq 0.1 \mathrm{mV}$ in two or more adjacent leads. Exclusion criteria included the following: advanced malignant tumor; history of myocardial infarction; acute inflammatory or infectious disease; decompensated liver disease; end-stage renal disease; severe heart failure (Killip IIIIV); history of coronary artery bypass; hypersensitivity to SBP;

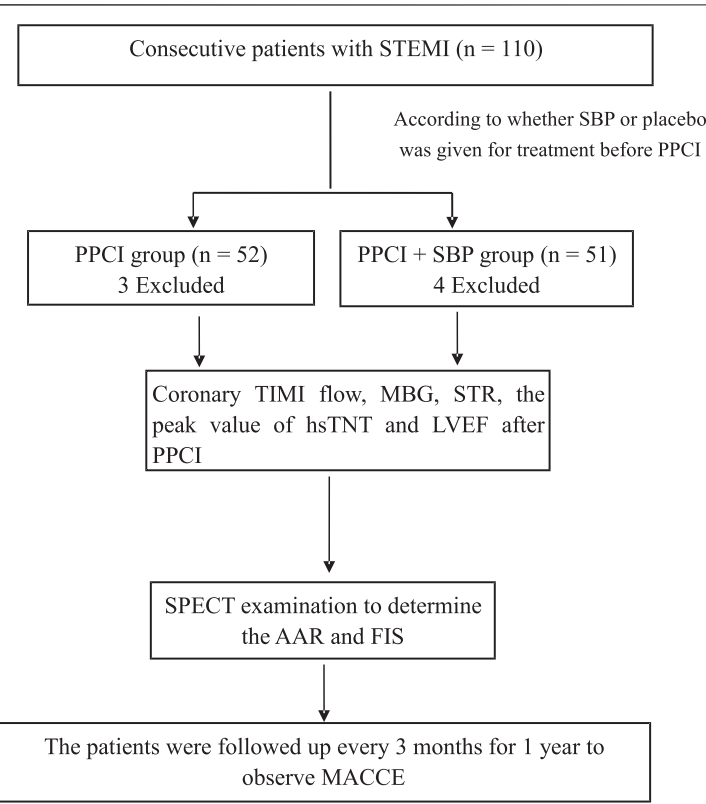

STEMI: ST-segment elevation myocardial infarction;

SBP: Shexiang Baoxin Pills;

PPCI: primary percutaneous coronary intervention;

STR: ST-segment resolution;

hsTNT: high-sensitivity troponin T;

LVEF: left ventricular ejection fraction;

SPECT: single-photon emission computed tomography;

AAR: area at risk of infarction;

FIS: final infarct size;

MACCE: major adverse cardiac and cerebrovascular events.

FIGURE 1 | Technical route.

failure to complete the study; and lack of follow-up. The selected patients were randomly divided into the PPCI group (52 cases, two patients had a history of myocardial infarction and one had severe heart failure (Killip III). A total of three patients were excluded from the standard) and the PPCI + SBP group (51 cases, 1 case had a history of myocardial infarction, 2 cases had severe heart failure (Killip III and Killip IV), 1 case had acute inflammation. A total of 4 cases were excluded) according to whether they were treated with SBP or not. All patients were treated with conventional medicine and PPCI implemented as early as possible $12 \mathrm{~h}$ from the onset of the symptoms according to the guidelines. SBP and placebo were given immediately after routine medication. The PPCI + SBP group was given two pills of SBP (Shanghai Hutchison Pharmaceuticals, $22.5 \mathrm{mg} / \mathrm{pill}$ ) immediately after admission as soon as possible, and then two pills each time, three times a day, during 1 month. The PPCI group was given placebo in the same way (Figure 1). The general baseline characteristics of all patients, including gender, age, smoking history, previous hypertension, diabetes, cerebral infarction history, coronary heart disease family history, admission heart rate, systolic blood pressure, diastolic blood pressure, creatinine, blood glucose, low-density lipoprotein, high-sensitivity troponin T (hsTNT), and myocardial ischemia time, were recorded. 
The research plan was approved by the ethics committee of Inner Mongolia Medical University. All patients provided written informed consent.

\section{Preoperative, Intraoperative, and Postoperative Medication}

All patients were given aspirin tablets $(300 \mathrm{mg})$, clopidogrel tablets ( $<75$ years old, $600 \mathrm{mg}$; or $\geq 75$ years old, $300 \mathrm{mg}$ ) or ticagrelor $180 \mathrm{mg}$ ( $<75$ years old) before PPCI. Intravenous heparin (100 IU $/ \mathrm{kg}$ ) was used. If the patient's thrombus load was heavy (thrombus grade $\geq 3)$, tirofiban $(10 \mu \mathrm{g} / \mathrm{kg})$ was given, then intravenous tirofiban $(0.15 \mu \mathrm{g} / \mathrm{kg} / \mathrm{min})$ was maintained for $24 \mathrm{~h}$ after which heparin was changed to $50-70 \mathrm{IU} / \mathrm{kg}$. Other medications included aspirin 75-100 mg (once a day) for life, clopidogrel $75 \mathrm{mg}$ (once a day) or ticagrelor $90 \mathrm{mg}$ (twice a day) (less than 75 years old) for at least 1 year. Other drugs, including angiotensin-converting enzyme inhibitor (ACEI), angiotensin receptor blockers (ARB), $\beta$-blockers, and statins, were used according to published guidelines.

\section{Experimental Instruments and Methods Biological Index Detection}

HsTNT was measured in peripheral venous blood of all patients at admission and every $6 \mathrm{~h}$ after admission until the peak of hsTNT appeared. HsTNT was determined by Elecsys 2010, Roche, Switzerland. Peripheral venous blood was drawn $24 \mathrm{~h}$ after admission, N-terminal pro-B type natriuretic peptide (NT-proBNP) was determined by Elecsys 2010, Roche, Switzerland. Blood routine examination, liver and kidney function, blood lipid and blood glucose, blood potassium, sodium and chlorine were measured conventionally.

\section{Process of Coronary Angiography and PPCI}

PPCI was performed through radial artery or femoral artery with a routine $6 \mathrm{~F}$ catheter, guide wire and balloon dilator. According to the thrombus load of the target vessel (thrombus grade $\geq 3$ ) Gibson et al. (2001), aspiration thrombectomy was used. Thrombolysis in myocardial infarction (TIMI) and myocardial blush grade (MBG) van 't Hof et al. (1998) were used to evaluate the coronary flow before and after operation. TIMI was divided into $0-3$ grades according to the filling condition of coronary artery contrast medium. Grade 0 indicated total occlusion without blood flow passing through, while grade 3 marked normal blood flow passing through the coronary artery (TheStudy Group (198, 1985). MBG was divided into 0-3 grades according to the myocardial development, with grade 0 indicating no myocardial development, and grade 3 denoting normal myocardial development (Gibson et al., 2001). Angiographic assessment was performed offline by two experienced observers blinded to the PPCI + SBP group or PPCI group, electrocardiographic and echocardiographic data, and the single-photon emission computed tomography (SPECT) imaging results. Disagreements between the two observers were resolved by joint evaluation of the data and discussion.

\section{ECG Examination}

ECG was reexamined $90 \mathrm{~min}$ after PPCI. The ST segment evaluation of ECG was also evaluated by two independent experienced professional doctors without knowledge of patient group assignment. The ST-segment resolution (STR) was recorded.

\section{Single-Photon Emission Computed Tomography}

All patients underwent SPECT (e.cam, Siemens) to evaluate the area at risk of infarction (AAR) $24 \mathrm{~h}$ to 7 days after PPCI. ${ }^{99}$ Technetiumsestamibi ( ${ }^{99} \mathrm{Tcm}-\mathrm{MIBI}$, Beijing Senke company or 401 atomic High Tech) $700 \mathrm{MBq}$ was injected intravenously into the patients first, and then detected with the double probe rotating gamma cameraautomatic direct analog computer (ADAC) with a high resolution flat hole collimator within $8 \mathrm{~h}$ after injection. The final infarct size (FIS) was evaluated by SPECT 30 days after PPCI (the patients rested in supine position for $30 \mathrm{~min}$, and then were detected by ADAC after intravenous injection of ${ }^{99} \mathrm{Tc}^{\mathrm{m}}$-MIBI $700 \mathrm{MBq}$ for $60 \mathrm{~min}$ ). AAR and FIS were mainly lower than normal pixels on the myocardium (AAR and FIS are expressed by the percentage of measured area in the left ventricular area and were calculated by quantitative perfusion software (QPS)). Two fully blinded operators, using an off line dedicated workstation, performed all measurements. Myocardial salvage (MS) was defined as the difference between infarct risk myocardium and infarct myocardial area (AAR-FIS). The salvage index (SI) was calculated as MS/AAR.

\section{Echocardiography}

All the selected patients were examined by two fully blinded operators using echocardiography (Vivid 7, GE) within 1 week, and left ventricular ejection fraction (LVEF) was measured using the Simpson method.

\section{CLINICAL END POINT EVENTS}

All patients were followed up by telephone or clinic every 3 months for at least 1 year. The main clinical end points were MACCE, including cardiac death, non-fatal myocardial infarction, new heart failure, new cerebral infarction, and transient cerebral ischemia attack (all the end points were confirmed by hospital medical records or contacting a competent doctor). Non-fatal reinfarction and new heart failure were diagnosed according to the existing diagnostic criteria. New cerebral infarction and transient ischemic attack were diagnosed by brain CT or MRI, and a neurologist was invited for diagnosis. MACCE records the occurrence according to the sequence of cardiac death $>$ non-fatal myocardial infarction $>$ new heart failure $>$ new cerebral infarction $>$ transient cerebral ischemia attack, so as to avoid repeated recording of cardiovascular and cerebrovascular events.

\section{STATISTICAL ANALYSIS}

SPSS17.0 (SPSS Inc., Chicago, Illinois, United States) was used for data analysis, and differences were considered statistically significant at $p<0.05$. The measurement data is expressed as mean \pm standard deviation $(\mathrm{x} \pm \mathrm{s})$. Independent $t$ test was used to evaluate the difference between the PPCI group and PPCI + SBP 
TABLE 1 | Comparison of clinical characteristics between PPCl + SBP and PPCl groups.

\begin{tabular}{|c|c|c|c|}
\hline- & PPCI Group (n = 52) & PPCI + SBP Group $(n=51)$ & $\mathbf{P}$ \\
\hline Age, years & $58.6 \pm 11.3$ & $57.2 \pm 9.6$ & 0.513 \\
\hline Gender (male), n (\%) & $42(80.8)$ & $43(84.3)$ & 0.636 \\
\hline History of Smoking, n (\%) & $34(65.4)$ & $34(66.7)$ & 0.891 \\
\hline History of hypertension, n (\%) & $23(44.2)$ & $22(43.1)$ & 0.769 \\
\hline History of diabetes, $\mathrm{n}(\%)$ & $8(15.4)$ & $7(13.7)$ & 0.811 \\
\hline History of cerebral infarction, n (\%) & $3(5.8)$ & $2(3.9)$ & 0.663 \\
\hline Admission Heart Rate, bpm & $70.5 \pm 14.1$ & $72.5 \pm 12.6$ & 0.451 \\
\hline Systolic blood pressure, mmHg & $126.4 \pm 24.7$ & $125.0 \pm 24.9$ & 0.778 \\
\hline Diastolic blood pressure, $\mathrm{mmHg}$ & $81.7 \pm 17.3$ & $79.8 \pm 15.4$ & 0.560 \\
\hline creatinine, umol/L & $78.3 \pm 19.5$ & $76.3 \pm 15.7$ & 0.555 \\
\hline Blood glucose, mmol/L & $7.9 \pm 3.2$ & $7.7 \pm 3.8$ & 0.781 \\
\hline Low density lipoprotein, mmol/L & $3.2 \pm 1.4$ & $3.2 \pm 0.8$ & 0.991 \\
\hline hsTNT at admission, ug/L & $1.10 \pm 2.17$ & $0.72 \pm 1.56$ & 0.314 \\
\hline The Peak Value of hsTNT, ug/L & $7.09 \pm 4.51$ & $5.54 \pm 3.23$ & 0.048 \\
\hline NT-proBNP after admission, pg/ml & $1,014.86 \pm 1,259.38$ & $567.02 \pm 988.82$ & 0.048 \\
\hline ST-segment resolution, \% & $63.30 \pm 22.14$ & $73.04 \pm 24.25$ & 0.036 \\
\hline LVEF, \% & $53.27 \pm 9.09$ & $56.53 \pm 7.42$ & 0.049 \\
\hline Myocardial ischemia time, $\mathrm{h}$ & $7.53 \pm 9.18$ & $6.21 \pm 2.93$ & 0.331 \\
\hline
\end{tabular}

HsTNT, high-sensitivity troponin T; LVEF, Left ventricular ejection fraction.

group. Variables of a non-normal distribution (determined by Kolmogorov-Smirnov test) are expressed as median and quartile ranges and were compared using Wilcoxon rank sum test. The counting data are expressed as the percentage composition ratio (\%) and were compared using the Chi-square test or Fisher's exact test. The Kaplan-Meier method was used to analyze MACCE cumulative events graphically.

\section{RESULTS}

\section{Comparison of Baseline and Clinical Characteristics}

Seven of the 110 STEMI patients were excluded. There was no significant difference in baseline characteristics between the two groups (Table 1). There was no significant difference in preoperative, intraoperative, and postoperative medication between the two groups $(p>0.05)$ (Table 2).

\section{Intraoperative and Postoperative Conditions \\ Intraoperative Conditions}

There was no significant difference in TIMI preoperative blood flow classification $(p=0.649$ ), TIMI postoperative blood flow classification ( $p=0.438)$, Number of diseased blood vessel ( $p=$ $0.417)$, target blood vessel $(p=0.603)$, thrombus aspiration $(p=$ $0.261)$, stent implantation $(p=0.414)$, and average number of stents $(p=0.81)$ between the two groups, while the MBG postoperative grade of the PPCI + SBP group was better than that of the PPCI group $(p=0.005)$ (Table 3).

Comparison of Postoperative Myocardial Reperfusion Assessed by STR and MBG

There was no significant difference in TIMI blood flow after PPCI between the PPCI + SBP group and PPCI group $(p=0.438)$
(Table 3). However, the MBG grade of the PPCI + SBP group was better than that of the PPCI group after PPCI $(p=0.005)$ (Table 3). In addition, the STR in the PPCI + SBP group was significantly higher than that in the PPCI group $(63.30 \pm 22.14 \%$ vs $73.04 \pm 24.25 \%$, respectively, $p=0.036$ ) (Table 1). These results confirm that SBP ameliorate myocardial reperfusion.

\section{I/R Injury Comparison Evaluated by SPECT}

There was no significant difference between the two groups regarding the AAR $[8.00 \%$ (3.00-25.75) vs $18.00 \%$ (5.25-29.00), respectively, $p=0.234]$, but the MS $[2.50 \%(0.125-9.750)$ vs $1.00 \%$ (-1.30-3.50), respectively, $p=0.023]$ and the SI $[27.95 \%$ $(0.00-45.00)$ vs $4.30 \%(-18.35-20.55)$, respectively, $p=0.006]$ were significantly improved in the PPCI + SBP group as compared to the PPCI group (Table 4). These indicate that SBP can alleviate I/R injury and rescue ischemic myocardium.

\section{Comparison of Myocardial Infarction Area by SPECT Evaluation and hsTNT}

Compared with the PPCI group, the peak value of hsTNT in the PPCI + SBP group decreased $(5.54 \pm 3.23 \mathrm{ug} / \mathrm{L}$ vs $7.09 \pm 4.51 \mathrm{ug} / \mathrm{L}$, respectively, $p=0.048$ ) (Table 1). SPECT showed that the FIS of the PPCI + SBP group decreased [6.50\% (3.00-15.00) vs $15.00 \%$ (4.00-29.50), respectively, $p=0.047$ ] as compared to PPCI group (Table 4), which mean that SPB can lower myocardial infarction area.

\section{Comparison of Cardiac Function Evaluated by LVEF and NT-proBNP}

Compared with the PPCI group, the PPCI + SBP group had higher LVEF (56.53 $\pm 7.42 \%$ vs $53.27 \pm 9.09 \%$, respectively, $p=0.049$ ), and lower NT-proBNP (567.02 \pm $988.82 \mathrm{pg} / \mathrm{ml}$ vs $1,014.86 \pm 1,259.38 \mathrm{pg} / \mathrm{ml}$, respectively, $p=$ 0.048) (Table 1). These results show that SBP improve cardiac function. 
TABLE 2 | Comparison of preoperative, intraoperative and postoperative medication between PPCl + SBP and PPCl.

\begin{tabular}{|c|c|c|c|}
\hline- & PPCI Group (n = 52) & PPCI + SBP Group $(n=51)$ & $\mathbf{P}$ \\
\hline heparin, n (\%) & $52(100.0)$ & $51(100.0)$ & 1.000 \\
\hline aspirin, n (\%) & $52(100.0)$ & $51(100.0)$ & 1.000 \\
\hline clopidogrel, n (\%) & 27 (51.9) & $19(37.3)$ & 0.134 \\
\hline ticagrelor, n (\%) & $26(50.0)$ & $32(62.7)$ & 0.192 \\
\hline tirofiban, n (\%) & $20(38.5)$ & $18(35.3)$ & 0.739 \\
\hline ACEI, n (\%) & $42(80.8)$ & $40(78.4)$ & 0.768 \\
\hline ARB, n (\%) & $5(9.6)$ & $5(9.8)$ & 0.974 \\
\hline$\beta$-blocker, n (\%) & $46(88.5)$ & $46(90.2)$ & 0.776 \\
\hline statins, n (\%) & 52 (100.0) & $51(100.0)$ & 1.000 \\
\hline
\end{tabular}

ACEl: angiotensin-converting enzyme inhibitor; ARB: angiotensin receptor blockers.

TABLE 3 | Comparison of intraoperative characteristics between $\mathrm{PPCl}+\mathrm{SBP}$ and $\mathrm{PPCl}$ groups.

\begin{tabular}{|c|c|c|c|}
\hline- & PPCI Group (n = 52) & $P P C I+S B P$ roup $(n=51)$ & $\mathbf{P}$ \\
\hline TIMI preoperative, n (\%) & - & - & 0.649 \\
\hline 0 & $28(53.8)$ & $22(43.1)$ & - \\
\hline 1 & $7(13.5)$ & $11(21.6)$ & - \\
\hline 2 & $10(19.2)$ & $11(21.6)$ & - \\
\hline 3 & 7 (13.5) & $7(13.7)$ & - \\
\hline TIMI postoperative, n (\%) & - & - & 0.438 \\
\hline 0 & $0(0.0)$ & $0(0.0)$ & - \\
\hline 1 & $2(3.8)$ & $1(2.0)$ & - \\
\hline 2 & $9(17.3)$ & $5(9.8)$ & - \\
\hline 3 & $41(78.8)$ & $45(88.2)$ & - \\
\hline MBG postoperative, n (\%) & - & - & 0.005 \\
\hline 0 & $0(0.0)$ & $0(0.0)$ & - \\
\hline 1 & $4(7.7)$ & $2(3.9)$ & - \\
\hline 2 & $30(57.7)$ & $15(29.4)$ & - \\
\hline 3 & $18(34.6)$ & $34(66.7)$ & - \\
\hline Number of diseased vessels, $n(\%)$ & - & - & 0.417 \\
\hline 1 & $11(21.2)$ & $14(27.5)$ & - \\
\hline 2 & $16(30.8)$ & 19 (37.3) & - \\
\hline 3 & $25(48.1)$ & $18(35.3)$ & - \\
\hline Target Vessel, n (\%) & - & - & 0.603 \\
\hline Left anterior descending artery & $23(44.2)$ & $27(52.9)$ & - \\
\hline circumflex artery & $5(9.6)$ & $3(5.9)$ & - \\
\hline right coronary artery & $24(46.2)$ & $21(41.2)$ & - \\
\hline aspiration thrombectomy, n (\%) & $14(26.9)$ & 19 (37.3) & 0.261 \\
\hline Average number of stents, $n(\%)$ & $1.33 \pm 0.73$ & $1.29 \pm 0.61$ & 0.81 \\
\hline stent implantation, n (\%) & $48(92.3)$ & $49(96.1)$ & 0.414 \\
\hline
\end{tabular}

TIMI, thrombolysis in myocardial infarction; MBG, myocardial blush grade.

\section{Clinical End Point Events}

All patients were followed through January 31, 2019. There was no significant difference between the two groups (556.9 \pm 191.5 days vs $483.1 \pm 205.0$ days, respectively, $p=0.062$ ). MACCE occurred in $10(9.7 \%)$ of all eligible patients: 7 cases (13.5\%) in the PPCI group and 3 cases $(5.9 \%)$ in the PPCI + SBP group. In the PPCI group, MACCE included 1 (1.9\%) cardiac death, 3 (5.8\%) non-fatal reinfarction, and 3 (5.8\%) new heart failure, while in the PPCI + SBP group, MACCE included 2 (3.9\%) non-fatal reinfarction and $1(2.0 \%)$ case of new heart failure. There was no significant difference in the incidence of MACCE ( 13.5 vs $5.9 \%, p=0.482$ ) between the PPCI group and the PPCI + SBP group (Figure 2), which mean decreased MACCE trend, but there was no statistical difference.

\section{DISCUSSION}

This study confirmed that SBP can reduce myocardial I/R injury, improve myocardial reperfusion, and reduce myocardial infarction area, as well as improve cardiac function, in patients with STEMI.

Reperfusion therapy can reduce the area of myocardial infarction as well as the incidence of heart failure and mortality in patients with STEMI, which is the most effective way to treat STEMI (Bulluck et al., 2016). I/R injury is induced by reperfusion therapy, which usually occurs in STEMI patients Hausenloy and Yellon (2013), and accounts for more than $50 \%$ of myocardial infarction area. This reduces the effect of reperfusion therapy on STEMI patients (Hausenloy and Yellon, 2015; Bulluck et al., 2016). 
TABLE 4 | Myocardial I/R injury and Final infarct size assessed by SPECT.

\begin{tabular}{|c|c|c|c|}
\hline- & $\mathrm{PPCl}$ group $(\mathrm{n}=52)$ & PPCI + SBP group $(n=51)$ & $\mathbf{P}$ \\
\hline Area at risk of infarction (AAR), LV\% & $\begin{array}{c}18.00(5.25-29.00) \\
(n=43)\end{array}$ & $\begin{array}{c}8.00(3.00-25.75) \\
(n=41)\end{array}$ & 0.234 \\
\hline Final Infarct Size (FIS), LV\% & $\begin{array}{c}15.00(4.00-29.50) \\
(n=52)\end{array}$ & $\begin{array}{c}6.50(3.00-15.00) \\
(n=51)\end{array}$ & 0.047 \\
\hline Myocardial salvage (MS), LV\% & $\begin{array}{c}1.00(-1.30-3.50) \\
(n=29)\end{array}$ & $\begin{array}{c}2.50(0.125-9.750) \\
(n=28)\end{array}$ & 0.023 \\
\hline Salvage index (SI), \% & $\begin{array}{c}4.30(-18.35-20.55) \\
(n=29)\end{array}$ & $\begin{array}{c}27.95(0.00-45.00) \\
(n=28)\end{array}$ & 0.006 \\
\hline
\end{tabular}

$L V \%, \%$ of the left ventricle; SPECT, single-photon emission computed tomography; I/R, ischemia reperfusion; $M S=A A R-F I S ; S I=M S / A A R$.

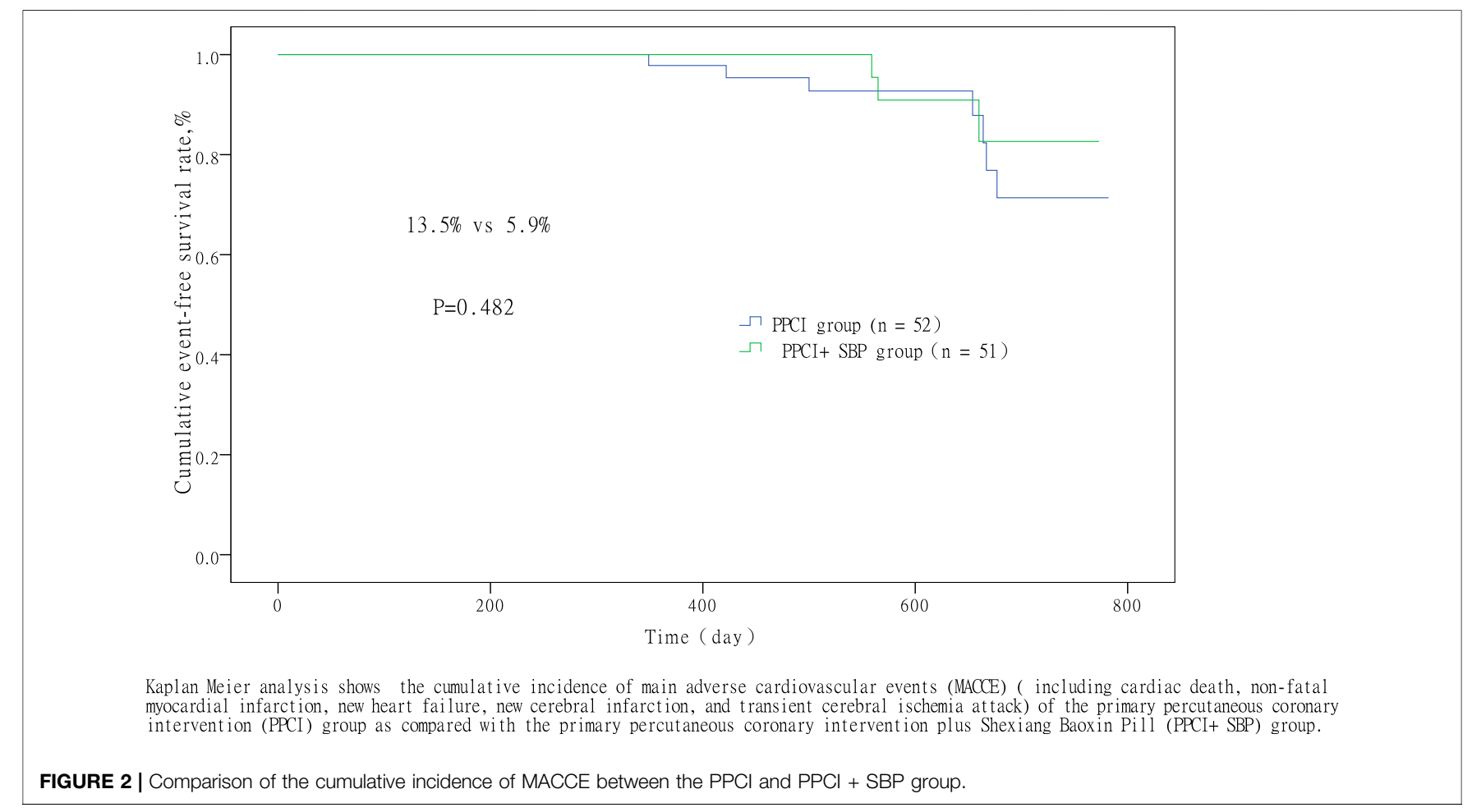

Myocardial infarction area is the main determinant of prognosis in patients with STEMI and is significantly related to cardiac function, cardiovascular adverse events, and cardiovascular death (Stone et al., 2016). Animal experimental studies have confirmed that many treatment measures can reduce myocardial I/R injury and myocardial infarction area (Hausenloy et al., 2017). However, there are few effective measures to reduce myocardial I/R injury and myocardial infarction area in STEMI patients, and clinical research results are inconsistently effective (Hausenloy and Bøtker, 2019). A meta-analysis involving nine randomized controlled trials (RCTs) and 1,220 patients found that remote ischemic conditioning (RIC) could reduce the myocardial infarction area (mean difference: $0.08 ; 95 \%$ confidence interval, 0.02-0.14) and increase the myocardial rescue index (mean difference: $-2.46 ; 95 \%$ confidence interval, -4.66 to -0.26) (McLeod et al., 2017). Man et al. conducted a meta-analysis that included 13 RCTs and 1756 patients. The results indicated that RIC reduced the creatine kinase-myocardial band $(\mathrm{CK}-\mathrm{MB})(p=0.0002)$, Troponin $\mathrm{T}$ (AUC, $p=0.003)$, and ST-segment resolution $\geq 70 \%(p=0.03)$ (Man et al., 2017). However, in the recent CONDI-2/ERICPPCI study, 5401 STEMI patients were enrolled and RIC was not found to reduce myocardial infarction area (Troponin $\mathrm{T}$ AUC, $p=0.48$ ) (Hausenloy et al., 2019). The METOCARDCNIC study found that early injection of metoprolol reduced myocardial infarction area in patients with STEMI (25.6 \pm 15.3 vs $32.0 \pm 22.2, p=0.012$ ) (Ibanez et al., 2013). GarcíaRuiz et al. also found that early injection of metoprolol in patients with anterior STEMI could reduce myocardial infarction area (22.9 vs $28.1, p=0.06$ ) (García-Ruiz et al., 2016). However, a multicenter RCT study of 683 STEMI patients did not confirm that metoprolol injection could reduce myocardial infarction area $(15.3 \pm 11.0 \%$ vs $14.9 \pm$ 
$11.5 \%, p=0.616$ ) (Li et al., 2019). Therefore, a single means treatment for myocardial I/R injury in STEMI patients may not be sufficient. Myocardial $I / R$ injury has a multimodal pathogenesis and thus treatment of $I / R$ injury may require targeting a variety of factors to achieve better results (Davidson et al., 2019; Li et al., 2019).

SBP is a traditional compound preparation composed of a variety of Chinese herbs Tian et al. (2018), diminishes the level of cardiac troponin $\mathrm{T}$ (cTNT) and cardiac troponin I (cTNI) in STEMI patients ( $\mathrm{Lu}$ et al. (2018), significantly decreases the frequency of angina in patients with stable CAD Ge et al. (2021), and lower the MACCE in patients with NSTE-ACS (Zhou et al., 2016). However, we confirmed that SBP was able to reduce the myocardial infarction area and relieve I/R injury assessed by SPECT that accurately assessed I/R injury and myocardial infarction area (Bøtker et al., 2010). It was found that Muscone, a component of the SBP heart protecting pill, can reduce myocardial remodeling and improve heart function of STEMI rats by inhibiting the inflammatory response and apoptosis while upregulating hypoxia-induced vascular endothelial growth factor (Du et al., 2018a; Du et al., 2018b). However, no RCTs have reported that SBP reduces myocardial remodeling and improves heart function in STEMI patients. Our present study verified that SBP can improve heart function in STEMI patients due to its effect on reducing myocardial infarction area and relieving myocardial I/R injury. Myocardial $\mathrm{I} / \mathrm{R}$ injury was decreased by inhibition of oxidative stress and apoptosis (Wu et al., 2011). Ginsenoside is the extract of Panax ginseng, which is a component of SBP; it can inhibit myocardial $\mathrm{I} / \mathrm{R}$ injury and reduce myocardial infarction area by inhibiting oxidative stress, the inflammatory response, and apoptosis (Zheng et al., 2017; Li et al., 2018; Li et al., 2020). Cinnamaniline is an extract of cinnamon and a component of SBP, which also limits myocardial I/R injury in rats with AMI. The mechanism of action is to inhibit the opening of the mitochondrial permeability transition pore (MPTP), inhibit calcium overload and reduce oxidative stress (Fancelli et al., 2014). Therefore, the components of SBP can reduce myocardial I/R injury and myocardial infarction area by inhibiting the opening of MPTP, calcium overload, inflammatory response, oxidative stress, and anti-apoptosis (Wu et al., 2011; Fancelli et al., 2014; Zheng et al., 2017; Li et al., 2018; Li et al., 2020). Xiang et al. found that SBP can treat AMI rats by acting on inflammatory response, abnormal energy metabolism, abnormal amino acid metabolism, and oxidative damage (Xiang et al., 2012). SBP may participate in angiogenesis in MI rats throug up-regulation of 20-hydroxyeicosatetraenoic acid Huang et al. (2017) and activating macrophages to regulate endothelial cell function and signal transduction pathways (Zhang et al., 2020). Therefore, SBP may also have multiple mechanisms of action on myocardial I/R injury. The therapeutic effect of SBP on STEMI patients may be due to the synergistic action of multiple drugs on multiple myocardial $\mathrm{I} / \mathrm{R}$ injury targets. The specific mechanism by which SBP acts is a main topic of future research. Meanwhile, Due to the defects of current pharmacological research methods, it is not enough to clearly demonstrate the mechanism of drugs with multiple targeted effects and molecular properties, which is also a challenge for this research at present.

In our study, SBP did not reduce MACCE (13.5 vs5.9\%, $p=$ 0.482). Possible reasons for this result are: 1) short follow-up time (Fancelli et al., 2014); and 2) the application of antiplatelet and statins may have diluted the curative effect of SBP(García-Ruiz et al., 2016). Our research also has some clinical limitations. The main reasons are too few samples, too short a follow-up time, and the fact that this was a single center study. A multicenter, randomized, double-blind, placebo-controlled clinical trial should be conducted to verify the therapeutic effect of SBP on I/R injury.

In conclusion, SBP can alleviate myocardial I/R injury, reduce myocardial infarction area, and improve cardiac function and myocardial reperfusion in patients with STEMI. If these results are confirmed in a large multicenter trial, SBP can become an effective measure against myocardial I/R injury, and may also become a part of the standard therapy for STEMI patients.

\section{DATA AVAILABILITY STATEMENT}

The original contributions presented in the study are included in the article/Supplementary Material, further inquiries can be directed to the corresponding author.

\section{ETHICS STATEMENT}

The studies involving human participants were reviewed and approved by Inner Mongolia Medical University. The patients/ participants provided their written informed consent to participate in this study.

\section{AUTHOR CONTRIBUTIONS}

HQ, SL, and ZL contributed to conception and design of the study. ZL organized the database. SL performed the statistical analysis. QH wrote the first draft of the manuscript. QH and ZL wrote sections of the manuscript. All authors contributed to manuscript revision, read, and approved the submitted version.

\section{FUNDING}

This study was supported by National Natural Science Foundation of China (81860053), the 2017 Autonomous Region Health and Family Planning Research Project of China (201702170), the Joint Project of Science and Technology Million Project of Inner Mongolia Medical University of China (YKD2016KJBW (LH)039) and the Independent Research Project of Ordos Central Hospital of China (EY2017001), Chinese Society of Integrated Chinese and Western MedicineHehuang Research Fund of China (HMP2005003 ${ }^{\mathrm{P}}$ ). 


\section{REFERENCES}

Bolognese, L., Carrabba, N., Parodi, G., Santoro, G. M., Buonamici, P., Cerisano, G., et al. (2004). Impact of Microvascular Dysfunction on Left Ventricular Remodeling and Long-Term Clinical Outcome after Primary Coronary Angioplasty for Acute Myocardial Infarction. Circulation 109 (9), 1121-1126. doi:10.1161/01.CIR.0000118496.44135.A7

Bøtker, H. E., Kharbanda, R., Schmidt, M. R., Bøttcher, M., Kaltoft, A. K., Terkelsen, C. J., et al. (2010). Remote Ischaemic Conditioning before Hospital Admission, as a Complement to Angioplasty, and Effect on Myocardial Salvage in Patients with Acute Myocardial Infarction: a Randomised Trial. Lancet 27 (9716), 727-734.

Bulluck, H., Yellon, D. M., and Hausenloy, D. J. (2016). Reducing Myocardial Infarct Size: Challenges and Future Opportunities. Heart 102 (5), 341-348. doi:10.1136/heartjnl-2015-307855

Davidson, S. M., Ferdinandy, P., Andreadou, I., Bøtker, H. E., Heusch, G., Ibáñez, B., et al. (2019). Multitarget Strategies to Reduce Myocardial Ischemia/ Reperfusion Injury: JACC Review Topic of the Week. J. Am. Coll. Cardiol. 73 (1), 89-99. doi:10.1016/j.jacc.2018.09.086

Du, Y, Ge, Y, Xu, Z, Aa, N., Gu, X, Meng, H., et al. (2018). Hypoxia-Inducible Factor 1 Alpha (HIF-1 $\alpha$ )/Vascular Endothelial Growth Factor (VEGF) Pathway Participates in Angiogenesis of Myocardial Infarction in Muscone-Treated Mice: Preliminary Study. Med. Sci. Monit. 24, 8870-8877. doi:10.12659/MSM.912051

Du, Y., Gu, X., Meng, H., Aa, N., Liu, S., Peng, C., et al. (2018). Muscone Improves Cardiac Function in Mice after Myocardial Infarction by Alleviating Cardiac Macrophage-Mediated Chronic Inflammation through Inhibition of NF-Kb and NLRP3 Inflammasome. Am. J. Transl. Res. 10 (12), 4235-4246.

Fancelli, D., Abate, A., Amici, R., Bernardi, P., Ballarini, M., Cappa, A., et al. (2014). Cinnamic Anilides as New Mitochondrial Permeability Transition Pore Inhibitors Endowed with Ischemia-Reperfusion Injury Protective Effect In Vivo. J. Med. Chem. 57 (12), 5333-5347. doi:10.1021/jm500547c

García-Ruiz, J. M., Fernández-Jiménez, R., García-Alvarez, A., Pizarro, G., GalánArriola, C., Fernández-Friera, L., et al. (2016). Impact of the Timing of Metoprolol Administration during STEMI on Infarct Size and Ventricular Function. J. Am. Coll. Cardiol. 67 (18), 2093-2104. doi:10.1016/ j.jacc.2016.02.050

Gbd 2015 Disease and Injury Incidence and Prevalence Collaborators (2018). Global, Regional, and National Incidence, Prevalence, and Years Lived with Disability for 310 Diseases and Injuries, 1990-2015: a Systematic Analysis for the Global Burden of Disease Study 2015. Lancet 388 (10053), $1545-1602$.

Ge, J.-B., Fan, W.-H., Zhou, J.-M., Shi, H.-M., Ji, F.-S., Wu, Y., et al. (2021). Efficacy and Safety of Shexiang Baoxin Pill (MUSKARDIA) in Patients with Stable Coronary Artery Disease: a Multicenter, Double-Blind, Placebo-Controlled Phase IV Randomized Clinical Trial. Chin. Med. J. (Engl). 134 (2), 185-192. doi: $10.1097 / \mathrm{cm} 9.0000000000001257$

Gibson, C. M., de Lemos, J. A., Murphy, S. A., Marble, S. J., McCabe, C. H., Cannon, C. P., et al. (2001). Combination Therapy with Abciximab Reduces Angiographically Evident Thrombus in Acute Myocardial Infarction: a TIMI 14 Substudy. Circulation 103 (21), 2550-2554. doi:10.1161/01.cir.103.21.2550

Hausenloy, D. J., Botker, H. E., Engstrom, T., Erlinge, D., Heusch, G., Ibanez, B., et al. (2017). Targeting Reperfusion Injury in Patients with ST-Segment Elevation Myocardial Infarction: Trials and Tribulations. Eur. Heart J. 38 (13), 935-941. doi:10.1093/eurheartj/ehw145

Hausenloy, D. J., and Bøtker, H. E. (2019). Why Did Remote Ischaemic Conditioning Not Improve Clinical Outcomes in Acute Myocardial Infarction in the CONDI-2/ERIC-PPCI Trial? Cardiovasc. Res. 115 (14), e161-e163. doi:10.1093/cvr/cvz242

Hausenloy, D. J., Kharbanda, R. K., Møller, U. K., Ramlall, M., Aarøe, J., Butler, R., et al. (2019). Effect of Remote Ischaemic Conditioning on Clinical Outcomes in Patients with Acute Myocardial Infarction (CONDI-2/ERIC-PPCI): a SingleBlind Randomised Controlled Trial. Lancet 394 (10207), 1415-1424. doi:10.1016/S0140-6736(19)32039-2
Hausenloy, D. J., and Yellon, D. M. (2013). Myocardial Ischemia-Reperfusion Injury: a Neglected Therapeutic Target. J. Clin. Invest. 123 (1), 92-100. doi:10.1172/JCI62874

Hausenloy, D. J., and Yellon, D. M. (2015). Targeting Myocardial Reperfusion Injury-The Search Continues. N. Engl. J. Med. 373 (11), 1073-1075. doi:10.1056/NEJMe1509718

Huang, F., Liu, Y., Yang, X., Che, D., Qiu, K., Hammock, B. D., et al. (2017). Shexiang Baoxin Pills Promotes Angiogenesis in Myocardial Infarction Rats via Up-Regulation of 20-HETE-Mediated Endothelial Progenitor Cells Mobilization. Atherosclerosis 263, 184-191. doi:10.1016/ j.atherosclerosis.2017.06.012

Ibanez, B., Macaya, C., Sánchez-Brunete, V., Pizarro, G., Fernández-Friera, L., Mateos, A., et al. (2013). Effect of Early Metoprolol on Infarct Size in STSegment-Elevation Myocardial Infarction Patients Undergoing Primary Percutaneous Coronary Intervention: the Effect of Metoprolol in Cardioprotection during an Acute Myocardial Infarction (METOCARDCNIC) Trial. Circulation 128 (14), 1495-1503. doi:10.1161/ CIRCULATIONAHA.113.003653

Li, J., Sun, D., and Li, Y. (2019). Novel Findings and Therapeutic Targets on Cardioprotection of Ischemia/Reperfusion Injury in STEMI. Curr. Pharm. Des. 25 (35), 3726-3739. doi:10.2174/1381612825666191105103417

Li, L., Pan, C. S., Yan, L., Cui, Y. C., Liu, Y. Y., Mu, H. N., et al. (2018). Ginsenoside Rg1 Ameliorates Rat Myocardial Ischemia-Reperfusion Injury by Modulating Energy Metabolism Pathways. Front. Physiol. 9, 78. doi:10.3389/ fphys.2018.00078

Li, L., Wang, Y., Guo, R., Li, S., Ni, J., Gao, S., et al. (2020). Ginsenoside Rg3Loaded, Reactive Oxygen Species-Responsive Polymeric Nanoparticles for Alleviating Myocardial Ischemia-Reperfusion Injury. J. Control. Release 317, 259-272. doi:10.1016/j.jconrel.2019.11.032

Lu, L., Sun, X., Chen, C., Qin, Y., and Guo, X. (2018). Shexiang Baoxin Pill, Derived from the Traditional Chinese Medicine, Provides Protective Roles against Cardiovascular Diseases. Front. Pharmacol. 9, 1161. doi:10.3389/ fphar.2018.01161

Man, C., Gong, D., Zhou, Y., and Fan, Y. (2017). Meta-analysis of Remote Ischemic Conditioning in Patients with Acute Myocardial Infarction. Sci. Rep. 7, 43529. doi:10.1038/srep43529

McLeod, S. L., Iansavichene, A., and Cheskes, S. (2017). Remote Ischemic Perconditioning to Reduce Reperfusion Injury during Acute STSegment-Elevation Myocardial Infarction: A Systematic Review and Meta-Analysis. J. Am. Heart Assoc. 6 (5), e005522. doi:10.1161/ JAHA.117.005522

Stone, G. W., Selker, H. P., Thiele, H., Patel, M. R., Udelson, J. E., Ohman, E. M., et al. (2016). Relationship between Infarct Size and Outcomes Following Primary PCI: Patient-Level Analysis from 10 Randomized Trials. J. Am. Coll. Cardiol. 67 (14), 1674-1683. doi:10.1016/j.jacc.2016.01.069

The TIMI Study Group (1985). The Thrombolysis in Myocardial Infarction (TIMI) Trial. Phase I Findings. N. Engl. J. Med. 312, 932-936. doi:10.1056/ NEJM198504043121437

Tian, P. P., Li, J., Gao, J., and Li, Y. (2018). Efficacy and Safety of the Shexiang Baoxin Pill for the Treatment of Coronary Artery Disease Not Amenable to Revascularisation: Study Protocol for a Randomised, Placebo-Controlled, Double-Blinded Trial. BMJ Open 8 (2), e018052. doi:10.1136/bmjopen-2017018052

van 't Hof, A. W., Liem, A., Suryapranata, H., Hoorntje, J. C., de Boer, M. J., and Zijlstra, F. (1998). Angiographic Assessment of Myocardial Reperfusion in Patients Treated with Primary Angioplasty for Acute Myocardial Infarction: Myocardial Blush Grade. Zwolle Myocardial Infarction Study Group. Circulation 97 (23), 2302-2306. doi:10.1161/ 01.cir.97.23.2302

Wu, Q., Li, H., Wu, Y., Shen, W., Zeng, L., Cheng, H., et al. (2011). Protective Effects of Muscone on Ischemia-Reperfusion Injury in Cardiac Myocytes. J. Ethnopharmacol. 138 (1), 34-39. doi:10.1016/j.jep.2011.08.009

Xiang, L., Jiang, P., Wang, S., Hu, Y., Liu, X., Yue, R., et al. (2013). Metabolomic Strategy for Studying the Intervention and the Synergistic Effects of the Shexiang Baoxin Pill for Treating Myocardial Infarction in Rats. Evid. Based Complement. Alternat. Med. 2013, 823121. doi:10.1155/ 2013/823121 
Xiang, L., Jiang, P., Zhan, C., Chen, Z., Liu, X., Huang, X., et al. (2012). The Serum Metabolomic Study of Intervention Effects of the Traditional Chinese Medicine Shexiang Baoxin Pill and a Multi-Component Medicine Polypill in the Treatment of Myocardial Infarction in Rats. Mol. Biosyst. 8 (9), 2434-2442. doi: $10.1039 / \mathrm{c} 2 \mathrm{mb} 25172 \mathrm{~h}$

Zhang, J., Cui, Q., Zhao, Y., Guo, R., Zhan, C., Jiang, P., et al. (2020). Mechanism of Angiogenesis Promotion with Shexiang Baoxin Pills by Regulating Function and Signaling Pathway of Endothelial Cells through Macrophages. Atherosclerosis 292, 99-111. doi:10.1016/j.atherosclerosis.2019.11.005

Zheng, Q., Bao, X. Y., Zhu, P. C., Tong, Q., Zheng, G. Q., and Wang, Y. (2017). Ginsenoside Rb1 for Myocardial Ischemia/Reperfusion Injury: Preclinical Evidence and Possible Mechanisms. Oxid Med. Cel. Longev. 2017, 6313625. doi:10.1155/2017/6313625

Zhou, Z., Shen, W., Yu, L., Xu, C., and Wu, Q. (2016). A Chinese Patent Medicine, Shexiang Baoxin Pill, for Non-ST-elevation Acute Coronary Syndromes: A Systematic Review. J. Ethnopharmacol. 194, 1130-1139. doi:10.1016/j.jep.2016.11.024
Conflict of Interest: The authors declare that the research was conducted in the absence of any commercial or financial relationships that could be construed as a potential conflict of interest.

Publisher's Note: All claims expressed in this article are solely those of the authors and do not necessarily represent those of their affiliated organizations, or those of the publisher, the editors and the reviewers. Any product that may be evaluated in this article, or claim that may be made by its manufacturer, is not guaranteed or endorsed by the publisher.

Copyright (C) 2021 Qin, Li and Liu. This is an open-access article distributed under the terms of the Creative Commons Attribution License (CC BY). The use, distribution or reproduction in other forums is permitted, provided the original author(s) and the copyright owner(s) are credited and that the original publication in this journal is cited, in accordance with accepted academic practice. No use, distribution or reproduction is permitted which does not comply with these terms. 